\title{
CARACTERIZAÇÃO FÍSICA E QUÍMICA DE FRUTOS DE MAMEY ${ }^{1}$
}

\author{
VIVIAN ELIAS NASCIMENTO², ANTONIO BALDO GERALDO MARTINS ${ }^{3}$, RONALDO HISSAYUKI HOJO ${ }^{4}$
}

RESUMO - O mamey (Pouteria sapota) é originário do México e América Central, sendo comum em Cuba, Norte da América do Sul e nas Índias Ocidentais. Porém somente após sua introdução na Flórida passou a ser mais conhecido e procurado, principalmente pelos latino-americanos. Para diversas fruteiras, a distinção entre variedades pode ser realizada com base em características dos frutos, permitindo a diferenciação dessas plantas. Diante disso, realizou-se o presente trabalho, verificando a possibilidade da distinção de plantas de mamey pertencentes à coleção do Banco Ativo de Germoplama, da FCAV, Câmpus de Jaboticabal-SP, através de características físicas e químicas dos frutos. Foram avaliadas: massa $(\mathrm{g})$, comprimento $(\mathrm{cm})$, diâmetro $(\mathrm{cm})$, rendimento de polpa (\%), acidez titulável (AT), sólidos solúveis (SS), ácido ascórbico (AA), pH e relação sólidos solúveis/acidez titulável de frutos de treze plantas de mamey. Com base nos resultados obtidos, pode-se concluir que existem diferenças significativas para todas as variáveis avaliadas entre as diferentes plantas de mamey, possibilitando a seleção de matrizes promissoras, para implantação de pomares comerciais.

Termos para indexação: Pouteria sapota; seleção; caracterização morfológica de frutos.

\section{PHYSICAL AND CHEMICAL CHARACTERIZATION OF MAMEY SAPOTA FRUITS}

ABSTRACT- The mamey sapota (Pouteria sapota) is original from Mexico and Central America, but it is quite common in Cuba, North of South America and the West Indies. However, only after its introduction in Florida, mamey has become well-known and sought especially by Latin Americans. The distinction among varieties can be done based on fruit characteristics, what permits the differentiation of these plants. In this context, the present research was accomplished, verifying the possibility to distinguish plants from mamey sapota belonging to the collection of the Active Germoplasm Bank, in the FCAV, Campus of Jaboticabal, São Paulo State, Brazil, by physical and chemical characteristics of the fruits. It was evaluated: weight, length, width, pulp percentage, soluble solid (SS), titratable acidity (TEA), ascorbic acid (AA), $\mathrm{pH}$ and SS/TEA ratio for thirteen plants of mamey sapota. There was a statistical difference between all the mamey sapota plants studied, making possible the selection of promising plants for the implantation of commercial orchards, and distinguishing the plants whose fruits present desirable characteristics for industrial use.

Index terms: Pouteria sapota; selection; fruit morphological characterization.

\section{INTRODUÇÃO}

O Brasil possui grande potencial para o cultivo de diversas espécies, pois apresenta uma diversidade muito grande de tipos de solos e climas que favorecem o cultivo de fruteiras tropicais, subtropicais e temperadas (Simão, 1998).

A procura pela diversificação de culturas proporcionou um aumento pelo interesse de cultivo e consumo de frutas exóticas. Este mercado é impulsionado pela busca por produtos diversificados, onde o aroma, sabor e valor nutritivo são valorizados.

O aproveitamento de espécies frutíferas exóticas reflete na oferta de novas alternativas de frutas frescas para consumo e matéria-prima para agroindústria, constituindo uma preciosa fonte de alimentos.

Dentre essas espécies exóticas, encontra-se o mamey [Pouteria sapota (Jacq) H.E. Moore \& Stearn], uma fruta pouco conhecida, mas, nos países de origem, tem certa importância como fruta atrativa e de alto valor alimentar. Pertence à família Sapotaceae, na qual se incluem aproximadamente 70 gêneros e mais de 800 espécies, dentre as quais se encontram o caimito
(Chrysophyllum caimito), o abiu (Pouteria caimito), o canistel (Pouteria campechiana) e o sapoti (Manilkara zapota) (Balerdi et al., 2005).

Originário do México e América Central, é bastante comum em Cuba, Norte da América do Sul e nas Índias Ocidentais. Porém, somente após sua introdução na Flórida passou a ser mais conhecido e procurado, principalmente pelos latino-americanos. Segundo Carrara et al. (2004), o Fairchild Tropical Botanic Garden (FTBG) possui a coleção mais representativa e de maior diversidade de genótipos de mamey dos Estados Unidos.

Balerdi et al. (2005) citam que os frutos de mamey são do tipo baga, formato ovóide ou elipsóide e com cálice persistente na base. A casca é espessa e de coloração amarronzada. A polpa, quando madura, pode ter coloração salmão, rosa, laranja, vermelha ou vermelho-marrom, é macia e suave. Tem sabor adocicado e único.

O mamey é considerado uma planta de clima tropical, tolerante às altas temperaturas, porém não tolerante ao frio e a solos mal drenados e inundados. Usualmente o mamey é propagado por sementes (Balerdi et al., 2005).

As árvores originadas de sementes começam a produzir

(Trabalho 027-08). Recebido em: 15-01-2008. Aceito para publicação em: 26-08-2008.

${ }^{2}$ Eng. Agr., Mestranda - Produção Vegetal - FCAV/UNESP - Bolsista CNPq. Via de acesso Prof. Paulo Donato Castellane, s/n. CEP: 14884-900, Jaboticabal-SP. E-mail: vivian_nascimento@ hotmail.com

${ }^{3}$ Eng. Agr., Dr., Professor Assistnte do Departamento de Produção Vegetal da FCAV/UNESP. E-mail: baldo@fcav.unesp.br

${ }^{4}$ Eng. Agr., Doutorando - Produção Vegetal - FCAV/UNESP 
a partir de 7 anos e, quando enxertadas, a frutificação dá-se entre 3 e 5 anos, chegando a produzir de 200 a 500 frutos por ano. Na Flórida, 15 variedades de Mamey já foram definidas, considerando-se as mais importantes a "Pantin", "Magana", "Tazumal" e "Pace" (Balerdi et al., 2005).

A caracterização de cultivares é uma etapa essencial em programas de certificação, melhoramento e conservação de germoplasma, pois permite o monitoramento da qualidade genética (International Board for Plant Genetic Resources, 1988; Zubrzycki, 1997), podendo ser realizada com base em diferenças morfológica das plantas, nas moléculas de proteínas e de DNA (Ferreira \& Grattapaglia, 1998). A escolha do método a ser empregado dependerá das condições técnicas existentes e da genética das espécies.

Para diversas fruteiras, a distinção entre variedades pode ser realizada com base em características dos frutos e folhas, permitindo a diferenciação dessas plantas (Galán Saúco \& Menini, 1989). Dessa forma, o objetivo do trabalho foi verificar a possibilidade da distinção de plantas de mamey pertencentes à coleção do Banco Ativo de Germoplama, da FCAV, Câmpus de Jaboticabal-SP, através de características dos frutos, com o intuito de selecionar as mais promissoras para cultivos comerciais ou programas de melhoramento.

\section{MATERIAL E MÉTODOS}

As avaliações foram realizadas em treze plantas de mamey, originadas de sementes, trazidas da Flórida em 1985, dentro do projeto de introdução e avaliação da FCAV, em colaboração com o Cenargen, Embrapa (Donadio et al., 1998). As plantas encontram-se na área experimental do Departamento de Produção Vegetal da Faculdade de Ciências Agrárias e Veterinárias - UNESP - Câmpus de Jaboticabal-São Paulo. As coordenadas geográficas do local são latitude $21^{\circ} 17^{\prime} 05^{\prime \prime} \mathrm{S}$ e longitude $48^{\circ} 17^{\prime} 09^{\prime \prime} \mathrm{W}$, com altitude de aproximadamente $590 \mathrm{~m}$. O clima da região, segundo classificação de Köeppen, é do tipo Cwa, subtropical, relativamente seco no inverno, com chuvas no verão, apresentando temperatura média anual de $22{ }^{\circ} \mathrm{C}$ e precipitação pluviométrica de $1.552 \mathrm{~mm}$. O solo é classificado como Latossolo Vermelho-Escuro, de textura média.

Os frutos foram coletados nas plantas e avaliados quando estavam aptos para consumo, ou seja, estádio de maturação maduro, e os tratamentos foram constituídos por plantas de mamey em função da alta variabilidade observada, resultante da segregação genética, as quais foram identificadas de acordo com sua disposição no pomar: P1, P2, P3, P4, P5, P6, P7, P8, P9, P11, P12, P13 e P14.

Foram coletados 50 frutos por planta e transportados para o Laboratório de Produtos Hortícolas do Departamento de Produção Vegetal da UNESP-FCAV, para as seguintes avaliações: Massa fresca média dos frutos, casca e semente: obtido com auxílio de uma balança digital com variação de $\pm 5 \mathrm{~g}$, sendo os dados expressos em g; comprimento e diâmetro dos frutos: determinados com auxílio de um paquímetro digital, expresso em centímetros; rendimento de polpa: obtido pela diferença entre massa do fruto e a casca mais a semente; acidez titulável (AT): obtida por titulação com NAOH 0,1 M, segundo a técnica do Instituto Adolfo Lutz (1985); pH: determinado no extrato do suco, utilizando-se de um medidor de pH de bolso modelo Checker; teor de sólidos solúveis (SS): determinado por leitura em refratômetro, segundo a metodologia da AOAC (1992); teor de ácido ascórbico: mg de ácido ascórbico por $100 \mathrm{~g}$ de polpa, obtida através da titulação com 2,6 diclorofenol indofenol de sódio (2,6 DINa); relação sólidos solúveis e acidez titulável (SS/AT).

Os dados foram submetidos à análise de variância, e as médias foram comparadas pelo teste Tukey, a 5\% de probabilidade, por meio do programa estatístico Sisvar (Ferreira, 2000). Foram realizadas análises de correlação entre todas as variáveis avaliadas, baseando-se na significância de seus coeficientes. A classificação de intensidade da correlação para $p$ $<0,01$ é considerada muito forte $(\mathrm{r} \pm 0,91 \mathrm{a} \pm 1,00)$, forte $(\mathrm{r} \pm 0,71$ $\mathrm{a} \pm 0,90)$, média $(\mathrm{r} \pm 0,51 \mathrm{a} \pm 0,70)$ e fraca $(\mathrm{r} \pm 0,31 \mathrm{a} \pm 0,50)$ (Guerra \& Liveira, 1999). Adicionalmente, foi realizada uma análise de agrupamento, com base nas características físicas e químicas dos frutos. A análise multivariada (UPGMA) e os dendogramas foram realizados utilizando o programa STATISTICA.

\section{RESULTADOS E DISCUSSÃO}

Observaram-se diferenças significativas para todas as variáveis avaliadas entre as treze plantas de mamey, confirmando a existência de variabilidade entre elas (Tabelas 1,2).

A. Características físicas dos frutos

$\mathrm{O}$ indivíduo $\mathrm{P} 3$ apresentou valores superiores aos demais em todas as variáveis avaliadas (Tabela 1), indicando seu potencial para ser selecionado como planta-matriz, porém não diferindo estatisticamente de $\mathrm{P} 13$ com relação a diâmetro e de P9, P11 e P14 no rendimento de polpa. A P8 apresentou menor comprimento e diâmetro de frutos, não diferindo de $\mathrm{P} 7$ em relação ao comprimento dos frutos e $\mathrm{P} 7$ apresentou menor massa fresca, diferindo estatisticamente somente de P3, P9, P12 e P13. Os frutos de mamey apresentaram peso médio de 454,38 $\mathrm{g}$, comprimento e diâmetro médio de 12,9 e 7,8 cm, respectivamente.

Neste trabalho, houve correlação positiva entre comprimento e diâmetro $\left(r_{S}=0,762, P=0.0022\right)$. A relação entre o comprimento e o diâmetro dos frutos pode ser utilizada para avaliar o formato dos frutos, sendo que os indivíduos avaliados apresentaram tendência ao formato ovóide. Outra correlação de interesse foi entre peso do fruto (massa fresca) e rendimento de polpa $\left(r_{s}=0,706, P=0.0076\right)$, ou seja, o peso do fruto é linearmente proporcional à quantidade de polpa; e entre diâmetro e peso, a correlação foi de $r_{S}=0,722(P=0.0052)$. O menor rendimento de polpa foi da $\mathrm{P} 4$, porém não diferindo estatisticamente dos indivíduos P1, P2, P5, P6, P7 e P12.

Pode-se observar pela análise de agrupamento a formação de três grupos principais, sendo um deles constituído apenas pelo indivíduo $\mathrm{P} 3$, que se apresentou $100 \%$ diferente dos demais (Figura 1). O segundo grupo foi constituído por um subgrupo abrangendo os materiais $\mathrm{P} 9$ e P13; e o terceiro grupo constituiuse de vários subgrupos, sendo representado pelos demais materiais analisados. Não foi verificado nenhum material semelhante, conforme já esperado, uma vez que as plantas foram 
originadas de sementes. No entanto, a dissimilaridade foi bem pequena, especialmente entre os materiais P1 e P4, P5 e P6, P11 e P14, P9 e P13, sendo a distância genética menor que 5\%.

B. Características químicas dos frutos

As análises dos teores de sólidos solúveis mostraram que o indivíduo $\mathrm{P} 7$ apresentou os maiores teores, porém diferindo estatisticamente somente de P1 e P4. O menor teor foi encontrado no indivíduo $\mathrm{P} 4$, que não diferiu estatisticamente de $\mathrm{P} 1, \mathrm{P} 3, \mathrm{P} 5$, P6, P8, P9, P11, P12, P13 e P14 (Tabela 2), mostrando que o teor de sólidos solúveis apresentou pouca variação entre as plantas de mamey. O valor médio observado de SS foi de 23,04, considerado um valor alto quando comparado com outras frutas, porém é similar ao sapoti $(25,98)$ (Araújo Neto, 2000). Altos teores de sólidos solúveis são importantes para a comercialização de frutas frescas e para o processamento. Em algumas frutas, como manga e laranja, os teores de sólidos solúveis são utilizados para indicar o ponto de maturação (Lopes et al., 2001). Essa variável poderá ser útil para a determinação do ponto de colheita de frutos de mamey, pois acredita-se que esses teores aumentem conforme a maturação.

Para a variável acidez titulável, o maior valor foi encontrado na P14 (0,5184\%), sendo que os demais indivíduos não variaram entre si, tendo o valor médio de $0,155 \%$ (Tabela 2 ). Araújo Neto (2000) também encontrou valores parecidos em sapoti $(0,12 \%)$. Baixos valores de acidez titulável, como registrado em todos os genótipos de mamey, são de grande importância para o consumo como frutas frescas (Seymour \& Tucker, 1996). Por outro lado, altos valores de acidez titulável são importantes para o processamento das frutas, reduzindo a necessidade de adicionar produtos artificiais, embora este não seja um fator limitante na seleção de genótipos (Nascimento et al.,1998).

Em relação ao $\mathrm{pH}$, as polpas apresentaram variação entre 6,59 e 7,40 (Tabela 2), com média de 6,87, ou seja, o mamey é um fruto pouco ácido que, de acordo com Stumbo (1965), é classificado por apresentar $\mathrm{pH}>4,5$, necessitando de maior controle no processamento, devido à possibilidade de crescimento de bactérias. Observou-se pouca variação entre os valores de pH, porém este é considerado por Leitão (1991) como um fator intrínseco ao alimento e exerce o maior efeito seletivo sobre a microflora apta a se desenvolver.
Para a relação sólidos solúveis/acidez titulável, o maior valor foi do indivíduo P7, não diferindo estatisticamente de P1 e P5 (Tabela 2). O teor de SS de P7 também superou os demais, e isso é importante para elevar essa relação. Salienta-se que os valores baixos geralmente indicam sabor ácido ou frutos azedos. De acordo com Pinto et al. (2003), a relação SS/AT constitui uma forma de avaliar a receptividade decorrente do sabor dos frutos melhor do que os seus teores de açúcares e de acidez, medidos isoladamente. Segundo Chitarra \& Chitarra (1990), a relação pode ser considerada como um critério de avaliação do "flavor", sendo que valores maiores podem significar melhoria de sabor, além de ser indicativo do nível de amadurecimento.

Os maiores teores de ácido ascórbico foram encontrados em P9 (36,98 mg.100 $\left.\mathrm{g}^{-1}\right)$ e P7 (33,68 mg.100 $\left.\mathrm{g}^{-1}\right)$, muito próximo ao valor descrito para a laranja, em média $40 \mathrm{mg}$. $100 \mathrm{~g}^{-1}$, porém inferiores aos teores médios encontrados em frutas como a acerola (1500 mg) e a goiaba (67 mg) (SBF, 1987). Os teores médios encontrados $\left(22,85 \mathrm{mg} .100 \mathrm{~g}^{-1}\right)$ são similares aos relatados pelo USDA (2005), de $20 \mathrm{mg} .100 \mathrm{~g}^{-1}$. Para as variáveis químicas estudadas, não se detectou nenhuma correlação significativa de interesse.

De acordo com o dendograma obtido pelo agrupamento dos materiais, segundo as variáveis químicas dos frutos (Figura 2), observa-se a formação de dois grandes grupos abrangendo vários subgrupos. A dissimilaridade entre os materiais P1 e P3; P11 e P12; P6 e P14 foi inferior a 10\%. Entre os grande grupos obtidos, é possível verificar distâncias genéticas de 35\% e 50\% aproximadamente, podendo ser consideradas baixas, pois Cavalcante et al. (2007) consideraram alta dissimilaridade valores maiores que $75 \%$.

Os genótipos avaliados apresentam variabilidade para todos os caracteres estudados, possibilitando a seleção de matrizes promissoras para implantação de pomares comerciais. Em geral, as plantas de mamey avaliadas apresentam boa adaptabilidade para as condições climáticas de Jaboticabal. Apesar do pequeno número de plantas avaliadas, elas demonstraram diversidade genética (Figuras 1 e 2), que foi definida por Daher et al. (1997) como a expressão da dissimilaridade entre dois indivíduos, enquanto o grau de parentesco entre linhas, cultivares ou populações pode ser entendido, de acordo com Dudley (1993), em termos de distância genética.

TABELA 1- Valores médios das características físicas de frutos de diferentes plantas de mamey. Jaboticabal, 2007.

\begin{tabular}{|c|c|c|c|c|}
\hline \multirow[t]{2}{*}{ Planta } & Comprimento & Diâmetro & \multirow{2}{*}{$\begin{array}{l}\text { Massa Fresca } \\
(\mathrm{g})\end{array}$} & \multirow{2}{*}{$\begin{array}{l}\text { Rendimento de Polpa } \\
(\%)\end{array}$} \\
\hline & \multicolumn{2}{|c|}{$(\mathrm{cm})$} & & \\
\hline P1 & $12,97 \mathrm{bcd}$ & 7,79 de & $386,53 \mathrm{de}$ & 66,02 cde \\
\hline P2 & $13,44 \mathrm{~b}$ & $7,72 \mathrm{de}$ & $435,2 \mathrm{de}$ & 67,22 bcde \\
\hline P3 & $17,31 \mathrm{a}$ & 9,50 a & $765,82 \mathrm{a}$ & 82,49 a \\
\hline P4 & $13,07 \mathrm{bcd}$ & $7,6 \mathrm{def}$ & $393,66 \mathrm{de}$ & $57,57 \mathrm{e}$ \\
\hline P5 & $12,16 \mathrm{cde}$ & 7,76 de & 374,76 de & $63,99 \mathrm{de}$ \\
\hline P6 & $13,46 \mathrm{~b}$ & $7,05 \mathrm{f}$ & 374,44 de & 67,05 bcde \\
\hline P7 & $11,12 \mathrm{ef}$ & 7,17 ef & $317,71 \mathrm{e}$ & $63,64 \mathrm{de}$ \\
\hline P8 & $10,19 \mathrm{f}$ & $5,77 \mathrm{~g}$ & 437,95 cde & $69,58 \mathrm{bcd}$ \\
\hline P9 & $13,62 \mathrm{~b}$ & $8,73 \mathrm{bc}$ & $561,47 \mathrm{bc}$ & $75,20 \mathrm{abc}$ \\
\hline P11 & $11,9 \mathrm{de}$ & $8,21 \mathrm{~cd}$ & $418,38 \mathrm{de}$ & 73,09 abcd \\
\hline P12 & $12,78 \mathrm{bcd}$ & $7,78 \mathrm{de}$ & 467,04 bcd & 64,75 de \\
\hline P13 & $13,16 \mathrm{bc}$ & $8,99 \mathrm{ab}$ & $567,08 \mathrm{~b}$ & $69,68 \mathrm{bcd}$ \\
\hline P14 & $13,24 \mathrm{bc}$ & 7,59 def & $406,81 \mathrm{de}$ & $77,14 \mathrm{ab}$ \\
\hline $\mathrm{CV}(\%)$ & 3,77 & 3,29 & 10,96 & 6,07 \\
\hline
\end{tabular}

Médias seguidas pela mesma letra na coluna não diferem entre si, pelo teste Tukey, ao nível de $5 \%$ de probabilidade. 
TABELA 2- Valores médios das análises químicas de frutos de diferentes plantas de mamey. Jaboticabal, 2007.

\begin{tabular}{|c|c|c|c|c|c|}
\hline Planta & $\begin{array}{c}\text { Sólidos solúveis } \\
\text { ( }{ }^{\circ} \text { Brix) }\end{array}$ & $\mathrm{pH}$ & $\begin{array}{c}\text { Acidez Titulável } \\
(\%)\end{array}$ & SS/AT & $\begin{array}{l}\text { Ac. Ascórbico } \\
\left(\mathrm{mg} \cdot 100 \mathrm{~g}^{-1}\right)\end{array}$ \\
\hline $\mathrm{P} 1$ & $21,00 \mathrm{bc}$ & $6,66 \mathrm{ab}$ & $0,09 \mathrm{c}$ & $268,46 a b c$ & $12,77 \mathrm{c}$ \\
\hline P2 & $26,08 \mathrm{ab}$ & $7,04 \mathrm{ab}$ & $0,17 b c$ & 179,22 bcde & $27,20 \mathrm{abc}$ \\
\hline P3 & $21,60 \mathrm{abc}$ & $6,64 \mathrm{ab}$ & $0,15 \mathrm{bc}$ & 153,13 cde & $13,35 \mathrm{c}$ \\
\hline $\mathrm{P} 4$ & $20,60 \mathrm{c}$ & $6,63 \mathrm{ab}$ & $0,12 \mathrm{c}$ & 179,95 bcde & $23,82 a b c$ \\
\hline P5 & $22,50 \mathrm{abc}$ & $6,7 \mathrm{ab}$ & $0,07 \mathrm{c}$ & $325,92 \mathrm{ab}$ & $27,91 \mathrm{abc}$ \\
\hline P6 & $23,80 \mathrm{abc}$ & $7,40 \mathrm{a}$ & $0,18 \mathrm{bc}$ & 142,70 cde & $30,75 \mathrm{abc}$ \\
\hline P7 & $26,40 \mathrm{a}$ & $7,18 \mathrm{ab}$ & $0,07 \mathrm{c}$ & 387,49 a & $33,68 \mathrm{ab}$ \\
\hline P8 & $23,40 \mathrm{abc}$ & $7,26 \mathrm{ab}$ & $0,15 \mathrm{bc}$ & $222,39 \mathrm{bcd}$ & $19,15 \mathrm{abc}$ \\
\hline P9 & $21,80 \mathrm{abc}$ & $6,85 \mathrm{ab}$ & $0,30 \mathrm{~b}$ & 76,92 dde & $36,98 \mathrm{a}$ \\
\hline P11 & $23,70 \mathrm{abc}$ & $6,59 \mathrm{~b}$ & $0,19 \mathrm{bc}$ & $139,93 \mathrm{cde}$ & $12,29 \mathrm{c}$ \\
\hline $\mathrm{P} 12$ & $22,80 \mathrm{abc}$ & $6,59 \mathrm{~b}$ & $0,18 \mathrm{bc}$ & $139,58 \mathrm{cde}$ & $13,18 \mathrm{c}$ \\
\hline P13 & $22,80 \mathrm{abc}$ & $6,94 \mathrm{ab}$ & $0,18 \mathrm{bc}$ & $139,53 \mathrm{cde}$ & $16,49 \mathrm{bc}$ \\
\hline $\mathrm{P} 14$ & $23,04 \mathrm{abc}$ & $6,88 \mathrm{ab}$ & $0,51 \mathrm{a}$ & $47,890 \mathrm{e}$ & $29,45 \mathrm{abc}$ \\
\hline CV(\%) & 10,45 & 5,14 & 40,42 & 37,83 & 37,88 \\
\hline
\end{tabular}

Médias seguidas pela mesma letra na coluna não diferem entre si, pelo teste Tukey, ao nivel de $5 \%$ de probabilidade.

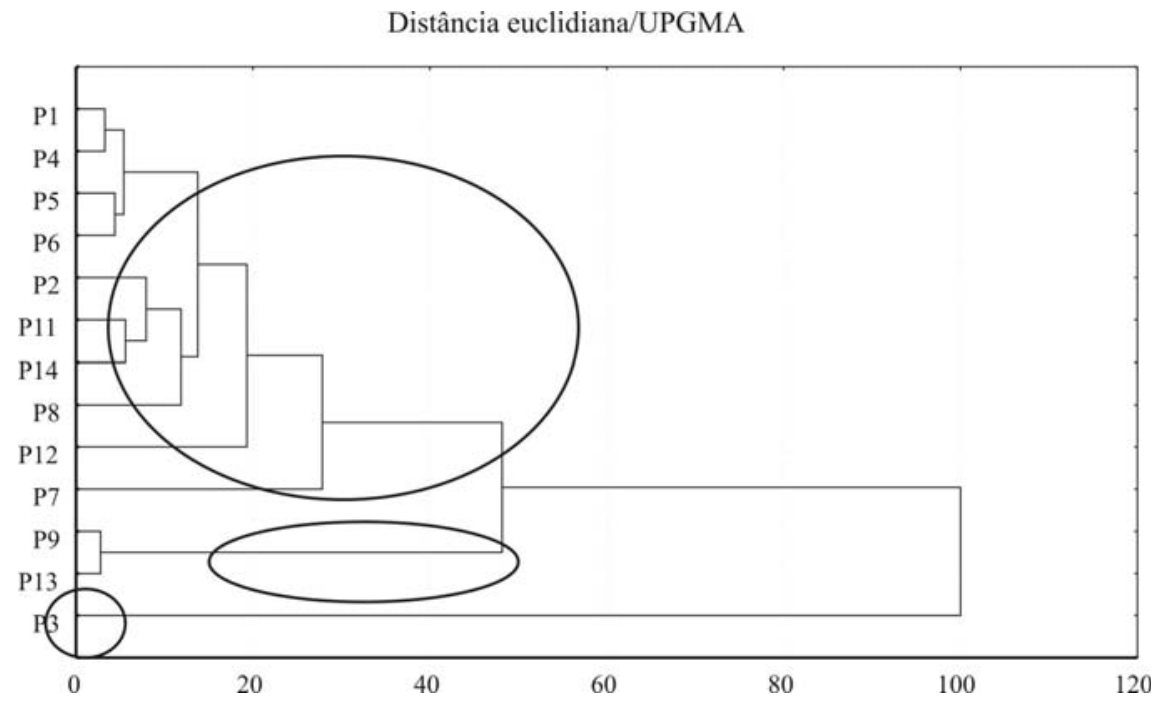

FIGURA 1- Análise de agrupamento das plantas de mamey, com base nas características físicas dos frutos. Jaboticabal, 2007.

Distância euclidiana/UPGMA

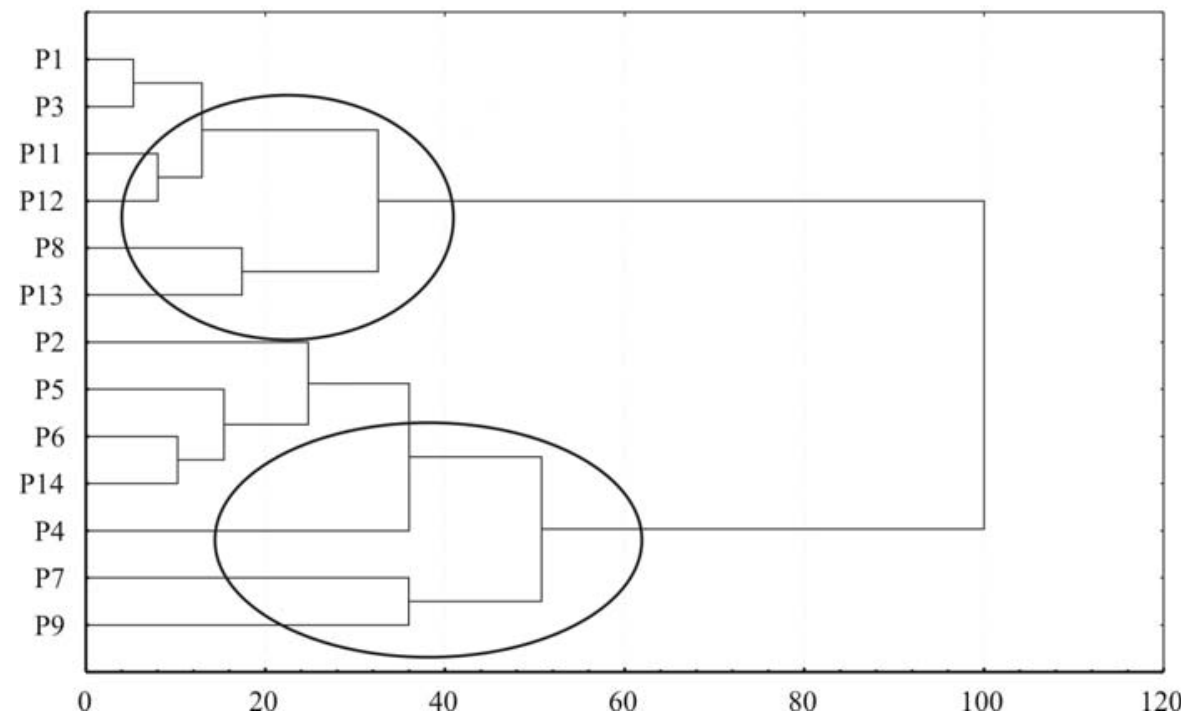

FIGURA 2 - Análise de agrupamento das plantas de mamey, com base nas características químicas dos frutos. Jaboticabal, 2007.

Rev. Bras. Frutic., Jaboticabal - SP, v. 30, n. 4, p. 953-957, Dezembro 2008 


\section{CONCLUSÕES}

1- As plantas de mamey que apresentam as melhores características dos frutos para processamento agroindustrial são:

- P3, apresentou maior rendimento de polpa.

- P7, apresentou elevado SS/AT e ácido ascórbico.

E o indivíduo P9 apresentou características importantes para o consumo como fruta fresca, pelo elevado teor de ácido ascórbico e um bom rendimento de polpa.

\section{REFERÊNCIAS}

ARAÚJO NETO, S.E. Desenvolvimento, maturação e determinação do ponto de colheita do sapoti (Manilkara achras (Mill.) Fosberg). 2000. 45 f. Dissertação (Mestrado em Fitotecnia) - Escola Superior de Agricultura de Mossoró, Mossoró, 2000.

AOAC - ASSOCIATION OF OFFICIAL ANALYTICAL CHEMISTRY. Official methods of analysis of the Association of Official Analytical Chemistry. $12^{\text {th }}$ ed. Washington, 1992. 1015 p.

BALERDI, C.F.; MAGUIRE, J.I. Mamey sapote growing in the florida home landscape. Gainsville, FL: Horticultural Sciences Department, University of Florida, 2005. Disponível em: http:// edis.ifas.ufl.edu/MG331.

CARRARA, S.; CAMPBELL, R.; SCHELL, R. Genetic variation among cultivated selections of mamey sapote. Proceedings of the Florida Horticultural Society, Winter Haven, v.117, p.195200.2004

CAVALCANTE, I.H.L.; BECKMANN, M.Z.; MARTINS, A.B.G.; CAMPOS, M.C.C. Preliminary selection of acerola genotypes in Brasil. Fruits, Paris, v. 62, p. 27-34, 2007.

CHITARRA, M.I.F.; CHITARRA, A.B. Pós-colheita de frutos e hortaliças. Lavras: FAEPE, 1990. 293p.

DAHER, R.F.; MORAES, C.F.; CRUZ, C.D. Seleção de caracteres morfológicos em capim-elefante (Pennisetum purpureum Schum.). Revista Brasileira de Zootecnia, Viçosa, v.26, p.247-259, 1997.

DONADIO, L. C.; NACHTIGAL, J. C.; SACRAMENTO, C. K. Frutas exóticas. Jaboticabal: FUNEP, 1998. v.1, 279 p.

DUDLEY, J.W. Molecular markers in plant improvement: manipulation of genes affecting quantitative traits. Crop Science, Madison, v.33, p.660-668, 1993.

FERREIRA, D.F. Análises estatísticas por meio do Sisvar para Windows versão 4.0. In: REUNIÃO ANUAL DA REGIÃO BRASILEIRA DA SOCIEDADE INTERNACIONAL DE BIOMETRIA, 45., 2000, São Carlos. Anais... São Carlos: UFSCAR, 2000. p. 255-258.

FERREIRA, M.E.; GRATTAPAGLIA, D. Introdução ao uso de marcadores moleculares em análise genética. 3. ed. Brasília: Embrapa-Cenargen, 1998. 220p.

GALÁN SAÚCO, V.; MENINI, U.G. Litchi cultivation. Roma: FAO Plant Production and Protection, 1989. 136p. (Paper, 83)

GUERRA, N.B.; LIVEIRA, A.V.S. Correlação entre o perfil sensorial e determinações físicas e químicas do abacaxi cv. Pérola. Revista Brasileira de Fruticultura, Jaboticabal, v. 21, n.1, p. 3235, 1999.

INSTITUTO ADOLFO LUTZ. Normas analíticas, métodos químicos e físicos para análise de alimentos. 3. ed. São Paulo, 1985. v. 1, 533 p.

INTERNATIONAL BOARD FOR PLANT GENETIC RESOURCES. Descriptors for citrus. Rome, 1988. 27p.

LEITÃO, M.F.F. Microbiologia de sucos, polpas e produtos ácidos: industrialização de Frutas. Campinas: ITAL, 1991. p.3352. (Manual Técnico, 8).

LOPES, R.; BRUCKNER, C.H.; CRUZ, C.D.; FREITAS, G.B. Repetibilidade de características do fruto de aceroleira. Pesquisa Agropecuária Brasileira, Brasília, v.36, p. 507-513, 2001.

NASCIMENTO T.B.; RAMOS J.D.; MENEZES J.B. Características físico-químicas do maracujá-amarelo (Passiflora edulis f. flavicarpa Deg.) produzido em diferentes épocas. Revista Brasileira de Fruticultura, Jaboticabal, v.20, p. 33-38. 1998.

PINTO, W. da S.; DANTAS, A.C.V.L.; FONSECA, A.A.O.; LEDO, C.A. da S.L.; JESUS, S.C. de; CALAFRANGE, P.L.P.; ANDRADE, E.M. Caracterização física, físico-química de frutos de genótipos de cajazeira. Pesquisa Agropecuária Brasileira, Brasília, v.38, n.9, p.1.059-1.066, 2003.

SEYMOUR, G.B.; TUCKER, G.A. Biochemistry of fruit ripening. London: Chapman \& Hall, 1996.

SIMÃO, S. Tratado de fruticultura. Piracicaba: FEALQ, 1998. 762p.

SBF - SOCIEDADE BRASILEIRA DE FRUTICULTURA. Valor nutricional de algumas frutas. Informativo SBF, Caçador, v.4, n.2, p.17, 1987.

STUMBO, C.R. Thermobacteriology in food processing. New York: Academic Press, 1965.

USDA - U.S. Department of Agriculture, Agricultural Research Service. Nutrient database for standard reference, Release 18. Nutrient Data Laboratory, 2005. Disponível em: http:// www.nal.usda.gov/fnic/foodcomp.

ZUBRZYCKI, H.M. Descritores básicos de diferentes órgãos de plantas cítricas para identificar mutantes, cultivares e híbridos. Corrientes: Instituto Nacional de Tecnologia Agropecuária, 1997. 14p 